\title{
Impact of casopitant, a novel NK-1 antagonist, on the pharmacokinetics of ondansetron and dexamethasone
}

\author{
Brendan Johnson • Laurel Adams • Emily Lu • \\ Ke Zhang • Peter Lebowitz • Christian Lates • \\ Robert Blum
}

Received: 31 July 2008 / Accepted: 22 December 2008 / Published online: 10 February 2009

(C) The Author(s) 2009. This article is published with open access at Springerlink.com

\begin{abstract}
Introduction Pharmacokinetic interactions between casopitant (a substrate and weak to moderate inhibitor of CYP3A), dexamethasone (a substrate and weak inducer of CYP3A), and ondansetron (a mixed CYP substrate) were evaluated in a two-part, three-period, single-sequence study in two groups of healthy subjects.

Materials and methods Part 1: subjects received oral casopitant (regimen A); oral dexamethasone and IV
\end{abstract}

Presented as an invited lecture at the Supportive Care in Cancer MASCC/ISOO 2008 International Symposium in Houston, Texas on June 26-28, 2008.

This work was sponsored by GlaxoSmithKline. C Lates and R Blum received funding from GlaxoSmithKline to conduct this study. All other authors were employees of GlaxoSmithKline.

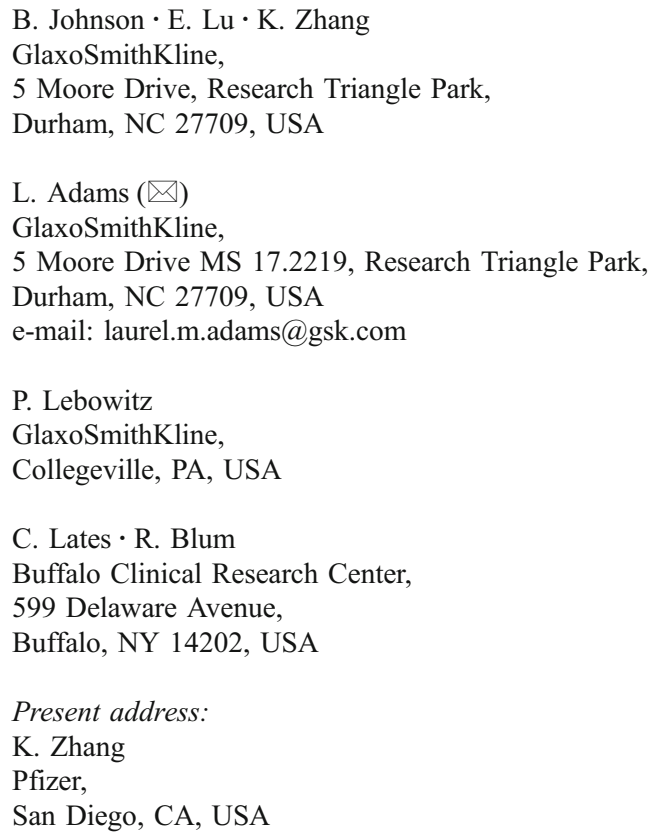

ondansetron (regimen B); and oral casopitant, a reduced dose of oral dexamethasone, and IV ondansetron (regimen C). Part 2: subjects received oral casopitant (regimen D); IV dexamethasone and oral ondansetron (regimen E); and oral casopitant, IV dexamethasone, and oral ondansetron (regimen F). Each regimen was separated by 14 days.

Results Casopitant AUC in regimen C was increased 28\% on day 1 but decreased $34 \%$ on day 3 compared to casopitant alone in regimen $\mathrm{A}$. When given with casopitant and ondansetron in regimen $\mathrm{C}$, dexamethasone AUC was $17 \%$ lower on day 1 , but similar on day 3 , compared to regimen B (representing dose-normalized increases in exposure of $39 \%$ and $108 \%$, respectively). Ondansetron exposure was equivalent in regimens B and C. Casopitant AUC in regimen F was similar to regimen D on days 1 and 3. Dexamethasone AUC increased 21\% when given with oral casopitant and oral ondansetron (regimen $\mathrm{F}$ compared to regimen $\mathrm{E}$ ). Ondansetron exposure was equivalent in regimens $\mathrm{E}$ and $\mathrm{F}$. Conclusion When repeat-dose oral dexamethasone is to be coadministered with oral casopitant, a reduction in dexamethasone dose may be considered; however, no change in casopitant dose is required. Ondansetron exposure was not affected by coadministration with casopitant.

Keywords Casopitant · Dexamethasone - Ondansetron · CINV

\section{Introduction}

Chemotherapy-induced nausea and vomiting (CINV) remains one of the most feared side effects of chemotherapy among cancer patients [25]. Several studies have reported a gap between healthcare providers' awareness of CINV and the actual incidence of CINV in patients, with most healthcare 
providers underestimating the actual prevalence of delayed nausea and vomiting $[9,15]$.

Failure to prevent CINV can negatively affect patient outcome by contributing to low adherence to and withdrawal from potentially effective chemotherapy $[20,21]$ and optimal prevention of CINV in the first cycle of chemotherapy can decrease the likelihood of CINV in subsequent cycles [4]. Furthermore, preventing CINV during all cycles lowers the probability of developing anticipatory CINV, a conditioned response that occurs prior to administration of chemotherapy [20,23].

Emesis is an autonomic reflex controlled by multiple neurotransmitter systems; two of the most important systems are the serotonin/5-hydroxytryptamine receptor $3\left(5-\mathrm{HT}_{3}\right)$ and substance P/neurokinin-1 receptor (NK-1) systems [13]. Blocking both the 5-HT 3 and NK-1 neurotransmitter receptors has been demonstrated to reduce CINV in patients receiving chemotherapy $[5,17,26]$. According to guidelines established by the Multinational Association for Supportive Care in Cancer, patients who will receive a moderately emetogenic chemotherapy (MEC) regimen should receive a $5-\mathrm{HT}_{3}$ receptor antagonist and dexamethasone, while patients who will receive a MEC regimen that includes anthracycline and cyclophosphamide or a highly emetogenic chemotherapy (HEC) regimen should receive a $5-\mathrm{HT}_{3}$ receptor antagonist, a corticosteroid, and an NK-1 receptor antagonist $[10,12,22]$.

A three-drug antiemetic regimen including a novel NK-1 receptor antagonist, casopitant, in combination with ondansetron and dexamethasone has recently demonstrated efficacy for the prevention of CINV in phase II [2] and phase III clinical trials $[3,8,11,24]$.

An important consideration when combining medications for therapeutic benefit is the potential for drug-drug interactions, particularly when the medications share metabolic pathways. Clinical and in vitro studies have demonstrated that the major enzymes involved in the metabolism of the NK-1 receptor antagonist casopitant are from the CYP3A family and that casopitant (and a major circulating metabolite) can result in weak to moderate inhibition of CYP3A.

Dexamethasone and ondansetron are commonly employed as part of an antiemetic regimen for the prevention of CINV. Dexamethasone is also metabolized by CYP3A and is a weak inducer of CYP3A after prolonged exposure or repeat-dose administration $[7,18]$. Ondansetron, much like other $5-\mathrm{HT}_{3}$ receptor antagonists, is metabolized by several CYP enzymes, primarily CYP1A2, CYP2D6, and CYP3A [6]. Consequently, there is a potential for casopitant to impact the pharmacokinetics of dexamethasone and ondansetron, and dexamethasone also may impact the pharmacokinetics of casopitant.

The purpose of the current study was to evaluate the potential pharmacokinetic interactions between casopitant, dexamethasone, and ondansetron using three drug regimens that may be employed for the prevention of CINV resulting from MEC or HEC. The dose of casopitant employed in the current study is the highest oral dose regimen currently under clinical investigation (employed in a phase III study examining the efficacy of casopitant for the prevention of CINV resulting from MEC [8]) and is a 3-day oral regimen of $150 \mathrm{mg}$ on day 1 , followed by $50 \mathrm{mg}$ on days 2 and 3 . Phase III studies (in both the HEC and MEC settings) also examined the efficacy of a 3-day regimen of casopitant where the day 1 oral dose was replaced by an IV dose of $90 \mathrm{mg}$ casopitant, selected to provide a similar casopitant exposure to the $150 \mathrm{mg}$ oral dose form. The results of the current study are therefore applicable to 3-day regimens of oral/oral or IV/oral casopitant, as well as a single-dose oral regimen consisting of $150 \mathrm{mg}$ casopitant on day 1 only.

\section{Materials and methods}

\section{Study population}

Healthy male and female subjects not undergoing chemotherapy, 18 to 55 years of age, with a body mass index of $\geq 19$ to $\leq 37 \mathrm{~kg} / \mathrm{m}^{2}$ and adequate organ function were eligible to enter the study. All females of childbearing potential were required to use birth control from 14 days before the first dose of study medication, throughout the study and for 14 days after the last dose of study medication. Exclusion criteria were designed to ensure that subjects were in good health and not receiving concomitant medications that may interfere with the pharmacokinetics of study medications.

The study protocol and informed consent documents were reviewed and approved by the institutional review board at the Buffalo Clinical Research Center. Subjects provided written consent prior to participation in the trial. All investigators were required to abide by Good Clinical Practices, International Conference on Harmonization guidelines, Declaration of Helsinki principles, and local laws and regulations.

\section{Study design}

This open-label, two-part, fixed-sequence study was conducted at one center in the USA. Subjects were only eligible to participate in one part of the study. Each part consisted of three treatment periods (regimens), with at least 14 days between each period. In part 1 , which was representative of a 3-day treatment regimen for the prevention of CINV resulting from HEC, subjects received oral casopitant in regimen $\mathrm{A}$, oral dexamethasone and IV ondansetron in regimen $\mathrm{B}$, and oral casopitant, a reduced 
dose of oral dexamethasone, and IV ondansetron in regimen $\mathrm{C}$ (Table 1). In part 2, which was representative of a 3-day treatment regimen that may be employed for the prevention of CINV resulting from $\mathrm{MEC}$, subjects received oral casopitant in regimen D, IV dexamethasone and oral ondansetron in regimen $\mathrm{E}$, and oral casopitant, IV dexamethasone, and oral ondansetron in regimen $\mathrm{F}$ (Table 1). All oral doses were taken with water after at least a $4 \mathrm{~h}$ fast, and when subjects were to receive multiple study medications, all doses were taken at the same time.

Blood samples for PK analysis were collected over a 24-h period on day 1 and day 3 of each treatment period at the following times: pre-dose and $0.25,0.5,1,1.5,2,3,5$, $8,12,16$, and $24 \mathrm{~h}$ post-dose. When dexamethasone was administered twice daily (part 1, regimen B), samples were collected pre-dose, and $0.25,0.5,1,1.5,2,3,5,8$, and $12 \mathrm{~h}$ after the morning and evening doses. This schedule allowed comparison of the $24 \mathrm{~h}$ exposure of dexamethasone after twice daily dosing of dexamethasone alone to that observed after once daily dosing when combined with casopitant. Adverse events (AEs) were reported and clinical laboratory values, ECGs, and vital signs were assessed.

\section{Pharmacokinetics}

Plasma samples were analyzed for casopitant, dexamethasone, and ondansetron using validated analytical methods based on protein precipitation, followed by HPLC/MS/MS analysis. Using a $50 \mu \mathrm{L}$ aliquot of human plasma the lower limit of quantification (LLQ) for casopitant was $1.50 \mathrm{ng} / \mathrm{mL}$ and the higher limit of quantification (HLQ) was 1,500 $\mathrm{ng} / \mathrm{mL}$; the LLQ for dexamethasone was $1.0 \mathrm{ng} / \mathrm{mL}$ and the HLQ was $500 \mathrm{ng} / \mathrm{mL}$. Using a $20 \mu \mathrm{L}$ aliquot of human plasma, the LLQ for ondansetron was $0.5 \mathrm{ng} / \mathrm{mL}$ and the HLQ was $500 \mathrm{ng} / \mathrm{mL}$. Quality control (QC) samples, prepared at three different analyte concentrations and stored with study samples, were analyzed with each batch of samples against separately prepared calibration standards. For the analysis to be acceptable, no more than one-third of the QC results were to deviate from the nominal concentration by more than $15 \%$ and at least $50 \%$ of the results from each QC concentration should be within $15 \%$ of nominal. The applicable analytical runs met all predefined run acceptance criteria.

Pharmacokinetic parameters were determined in parts 1 and 2 on both day 1 and 3 in periods 1,2, and 3 using standard non-compartmental methods (WinNonlin, version 4.1, Pharsight Corp, Mountain View CA, USA). AUC was calculated using the linear up-log down method, with $\mathrm{AUC}(0-\tau)$ being calculated for repeat-dose study medications [AUC(0-24) was calculated for twice daily dexamethasone] and $\operatorname{AUC}(0-\infty)$ being calculated for single-dose IV ondansetron or dexamethasone administration. Cmax, tmax, and half-life were also determined.

\section{Statistics}

For the assessment of the interactions between casopitant, dexamethasone, and ondansetron pharmacokinetics, $\log (\mathrm{e})$ transformed AUC and Cmax parameters were statistically analyzed by performing an analysis of variance (ANOVA) for each analyte (casopitant, dexamethasone, or ondansetron), day (1 or 3 ) and part (1 or 2) separately. The ANOVA used a mixed-effects model with subject as a random effect and treatment as a fixed effect. For assessment of the effect of dexamethasone and/or ondansetron on casopitant pharmacokinetics, regimen A or D was the reference treatment and regimen $\mathrm{C}$ or $\mathrm{F}$ was the test treatment, respectively. For assessment of the effect of casopitant on dexamethasone and ondansetron pharmacokinetics, regimen $\mathrm{B}$ or $\mathrm{E}$ was the reference treatment and regimen $\mathrm{C}$ or $\mathrm{F}$ was the test treatment, respectively. Point estimates and $90 \%$ confidence intervals for the differences of interest were constructed using the residual variance for each part. Point and interval estimates were then exponentially back transformed to

Table 1 Casopitant, dexamethasone, and ondansetron dosing regimens for parts 1 and 2

\begin{tabular}{llll}
\hline Part & Regimen & Day 1 & Days 2 and 3 \\
\hline 1 (HEC regimen) & Regimen A & $150 \mathrm{mg}$ oral casopitant once daily & $50 \mathrm{mg}$ oral casopitant once daily \\
& Regimen B & $20 \mathrm{mg}$ oral dexamethasone once daily & $8 \mathrm{mg}$ oral dexamethasone twice daily \\
& Regimen C & $150 \mathrm{mg}$ IV ondansetron single-dose & $50 \mathrm{mg}$ oral casopitant once daily \\
& & $12 \mathrm{mg}$ oral dexamethasone once daily & $8 \mathrm{mg}$ oral dexamethasone once daily \\
2 (MEC regimen) & $32 \mathrm{mg} \mathrm{IV} \mathrm{ondansetron} \mathrm{single-dose}$ & $50 \mathrm{mg}$ oral casopitant once daily \\
& Regimen D & $150 \mathrm{mg}$ oral casopitant once daily & $8 \mathrm{mg}$ oral ondansetron twice daily \\
& Regimen E & $8 \mathrm{mg} \mathrm{IV} \mathrm{dexamethasone} \mathrm{single-dose}$ & $50 \mathrm{mg}$ oral casopitant once daily \\
& Regimen F & $150 \mathrm{mg}$ oral ondansetron twice daily & $8 \mathrm{mg}$ oral ondansetron twice daily \\
\end{tabular}


Table 2 Demographic characteristics of enrolled subjects

\begin{tabular}{lll}
\hline & Part 1 $(n=23)$ & Part 2 $(n=21)$ \\
\hline $\begin{array}{l}\text { Age, years } \\
\text { Median (min-max) }\end{array}$ & $27.0(20-54)$ & $29.0(18-55)$ \\
$\begin{array}{l}\text { Gender, } n(\%) \\
\text { Female }\end{array}$ & $7(30)$ & $6(29)$ \\
Male & $16(70)$ & $15(71)$ \\
$\begin{array}{l}\text { Weight, kg } \\
\text { Median (range) }\end{array}$ & $78.90(61.2-100.2)$ & $75.30(50.4-103.4)$ \\
$\begin{array}{l}\text { BMI, kg/m } \\
\text { Median (range) }\end{array}$ & $24.40(20.0-32.3)$ & $25.70(20.2-32.7)$ \\
$\begin{array}{l}\text { Ethnicity, } n(\%) \\
\text { Hispanic or Latino }\end{array}$ & $2(9)$ & 0 \\
$\begin{array}{l}\text { Not Hispanic or Latino } \\
\text { Race }\end{array}$ & $21(91)$ & $21(100)$ \\
African American/African & $6(26)$ & $10(48)$ \\
$\quad$ heritage & & 0 \\
Asian-East Asian heritage & $1(4)$ & $11(52)$ \\
White-White/Caucasian/ & $16(70)$ & \\
$\quad$ European heritage & &
\end{tabular}

construct point and interval estimates for the ratios of interest. As comparisons of dexamethasone exposure in regimens $\mathrm{A}$ and $\mathrm{C}$ were made with different dexamethasone dose levels, dose-normalized comparisons were also made, where the dose-normalized fold-change was determined by the ratio of the two dose levels (per day) multiplied by the point estimate.

A sufficient number of subjects were enrolled to ensure that at least 18 evaluable subjects completed each part of the study. Based on the within-subject coefficients of variation for casopitant, dexamethasone, and ondansetron AUC and Cmax observed in previous studies $(34.2 \%$ being the highest value), a sample size of 18 subjects should result in the lower and upper bounds of the $90 \%$ CI to be within $22 \%$ of the point estimates. This calculation was based on a symmetric two-tailed proce- dure on the $\log (\mathrm{e})$ scale and a type I error rate of $5 \%$ in each tail.

\section{Results}

A total of 44 subjects were enrolled in the study, 23 in part 1 and 21 in part 2 . The majority of subjects in part 1 were White, while approximately half of the subjects in part 2 were African American. Demographic characteristics were otherwise similar in parts 1 and 2 and are summarized in Table 2. Forty subjects completed the study (four subjects withdrew during the conduct of part 1 , none due to adverse events).

\section{Pharmacokinetics}

\section{Part 1: highly emetogenic chemotherapy regimen}

Casopitant Single-dose administration (day 1) showed that coadministration of $150 \mathrm{mg}$ oral casopitant with $12 \mathrm{mg}$ oral dexamethasone (reduced-dose dexamethasone regimen compared to the standard dose of $20 \mathrm{mg}$ ) and $32 \mathrm{mg}$ IV ondansetron resulted in a $28 \%$ increase in mean casopitant plasma $\mathrm{AUC}(0-\tau)$ when compared to casopitant administered alone (Table 3 and Fig. 1). After 3 days coadministration of oral casopitant (150 mg day 1, $50 \mathrm{mg}$ days 2 and 3 ) and the reduced-dose regimen of oral dexamethasone (12 $\mathrm{mg}$ day 1 , $8 \mathrm{mg}$ once daily days 2 and 3$)$, casopitant $\operatorname{AUC}(0-\tau)$ and Cmax decreased by $34 \%$ and $18 \%$, respectively, compared to 3 days of administration of casopitant alone (Table 3 ).

Dexamethasone On day 1, coadministration $12 \mathrm{mg}$ oral dexamethasone and $150 \mathrm{mg}$ oral casopitant and $32 \mathrm{mg}$ IV ondansetron resulted in a lower mean dexamethasone AUC $(0-\tau)$ and Cmax, by $17 \%$ and $35 \%$, respectively, when compared to administration of $20 \mathrm{mg}$ oral dexamethasone

Table 3 Comparison of casopitant, dexamethasone, and ondansetron AUC and Cmax from parts 1 and 2

\begin{tabular}{lllll}
\hline Part & Analyte (regimen comparison) & Day & AUC Geometric mean ratio (90\% CI) & Cmax Geometric mean ratio (90\% CI) \\
\hline 1 (HEC regimen) & Casopitant (Reg C: Reg A) & 1 & $1.28(1.14,1.44)$ & $1.06(0.95,1.2)$ \\
& & 3 & $0.66(0.598,0.73)$ & $0.82(0.74,0.91)$ \\
& Dexamethasone (Reg C: Reg B) & 1 & $0.83(0.77,0.91)$ & $0.65(0.59,0.73)$ \\
& & 3 & $1.04(0.97,1.11)$ & $0.96(1.20,1.57)$ \\
& Ondansetron (Reg C: Reg B) & 1 & $1.09(1.02,1.16)$ & NA \\
& & 3 & NA & $1.27(1.16,1.39)$ \\
2 (MEC regimen) & Casopitant (Reg F: Reg D) & 1 & $1.16(1.07,1.26)$ & $0.90(0.81,1.01)$ \\
& & 3 & $0.92(0.83,1.01)$ & $0.95(0.88,1.04)$ \\
& Dexamethasone (Reg F: Reg E) & 1 & $1.21(1.14,1.28)$ & NA \\
& & 3 & NA & $0.96(0.85,1.08)$ \\
& Ondansetron (Reg F: Reg E) & 1 & $1.02(0.94,1.11)$ & $1.00(0.93,1.08)$ \\
\hline
\end{tabular}



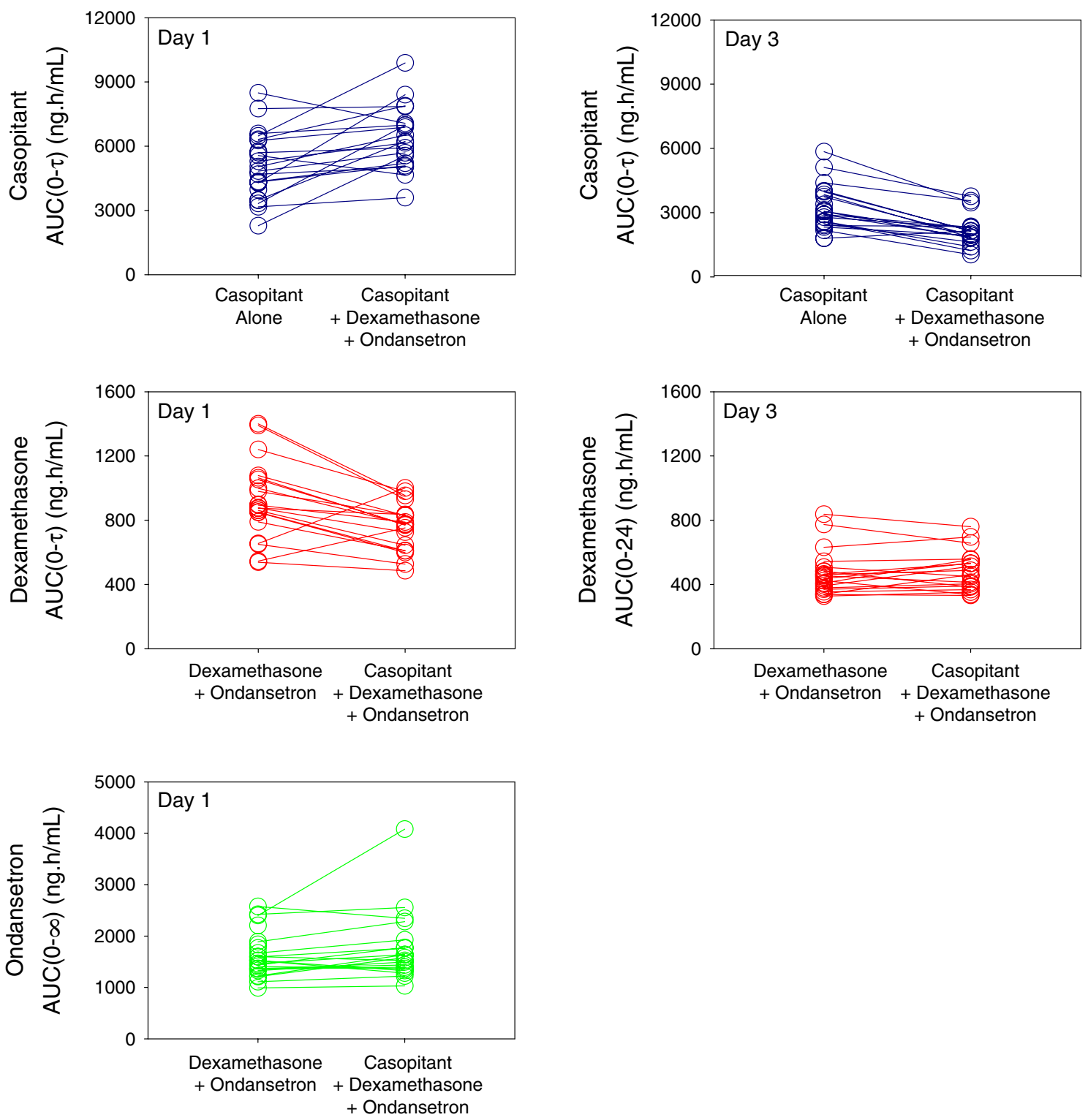

Fig. 1 Changes in individual casopitant (top), dexamethasone (middle), and ondansetron (bottom) AUC parameters after administration of casopitant alone, dexamethasone + ondansetron, and casopitant + dexamethasone + ondansetron, from part 1 (HEC regimen)

and $32 \mathrm{mg}$ IV ondansetron (Table 3 and Fig. 1). Dosenormalization of the pharmacokinetic parameters showed that casopitant increased the $\mathrm{AUC}(0-\tau)$ of oral dexamethasone by $39 \%$. After 3 days of coadministration, the $24 \mathrm{~h}$ AUC resulting from $8 \mathrm{mg}$ once daily oral dexamethasone combined with $50 \mathrm{mg}$ oral casopitant was similar to that resulting from $8 \mathrm{mg}$ twice daily oral dexamethasone alone, and represented a dose-normalized increase in dexamethasone exposure of $108 \%$. The mean increase in the Cmax of $8 \mathrm{mg}$ oral dexamethasone on day 3 was $37 \%$.

Ondansetron Plasma exposures of $32 \mathrm{mg}$ IV ondansetron were not affected by coadministration with casopitant (Table 3 and Fig. 1).

\section{Part 2: moderately emetogenic chemotherapy regimen}

Casopitant Single-dose administration (day 1) showed coadministration of a single-dose of $150 \mathrm{mg}$ oral casopitant with $8 \mathrm{mg}$ IV dexamethasone and $8 \mathrm{mg}$ twice daily oral ondansetron resulted in an increase in mean plasma casopitant $\mathrm{AUC}(0-\tau)$ and $\mathrm{Cmax}$ of $16 \%$ and $27 \%$, respectively (Table 3 and Fig. 2). The exposure of $50 \mathrm{mg}$ oral casopitant was not affected by 3 days of coadministration with $8 \mathrm{mg}$ twice daily oral ondansetron.

Dexamethasone The day 1 single-dose IV AUC $(0-\infty)$ of dexamethasone was increased by $21 \%$ when coadministered with $150 \mathrm{mg}$ oral casopitant and $8 \mathrm{mg}$ twice daily 

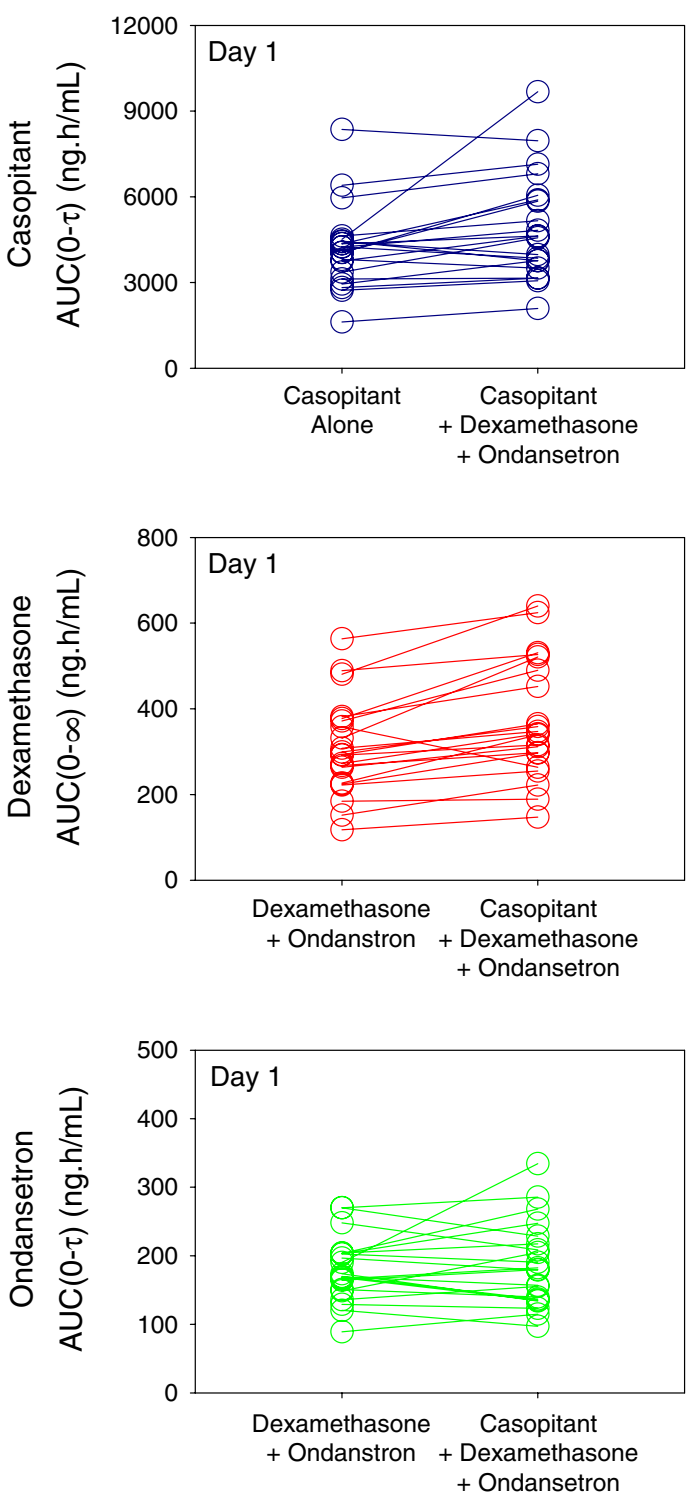

Fig. 2 Changes in individual casopitant (top), dexamethasone (middle), and ondansetron (bottom) AUC parameters after administration of casopitant alone, dexamethasone + ondansetron, and

ondansetron, but there was no change in dexamethasone Cmax (Table 3 and Fig. 2).

Ondansetron The day 1 and day 3 oral ondansetron $\operatorname{AUC}(0-\tau)$ and $\mathrm{Cmax}$ were not affected by coadministration with casopitant (Table 3 and Fig. 2).

\section{Safety}

The most commonly reported AEs were headache and dizziness. In part 1 , subjects who received oral dexamethasone and IV ondansetron demonstrated a virtually identical frequency of AEs, $58 \%$ and $59 \%$, respectively, in the
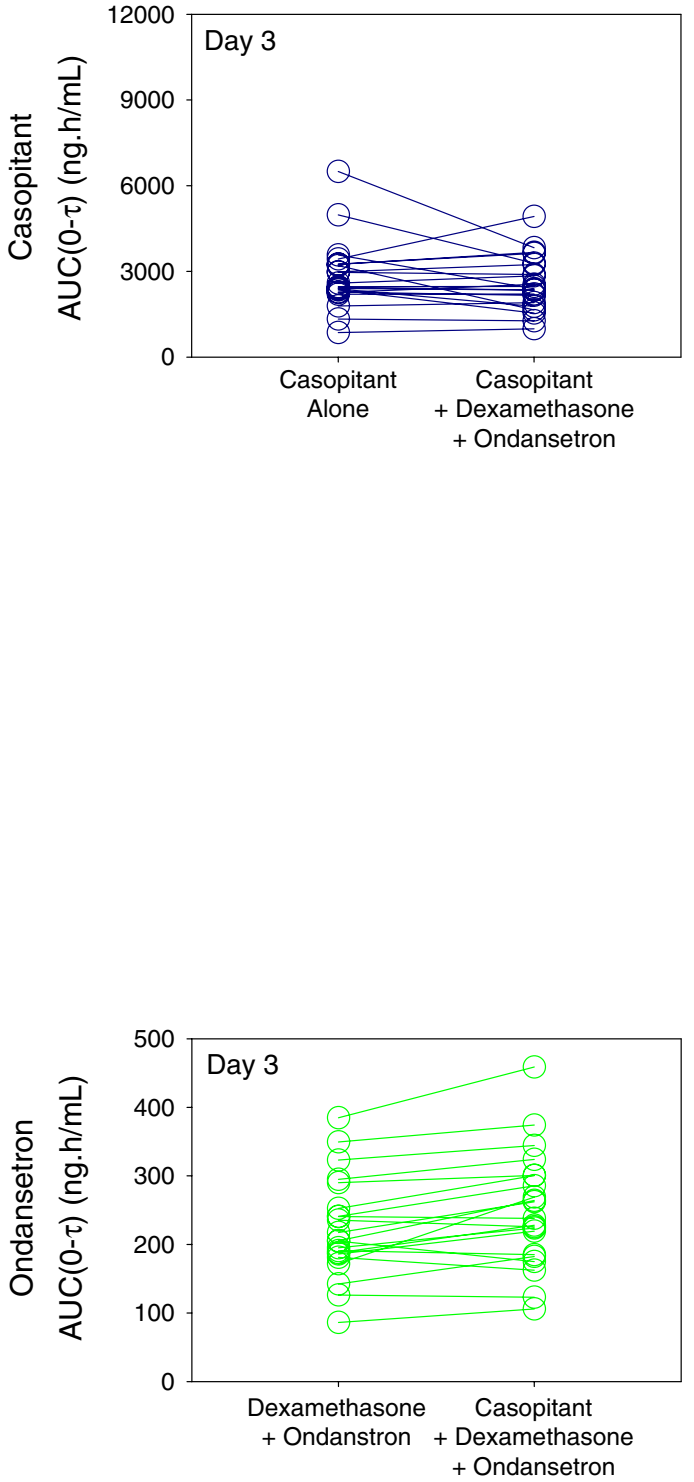

casopitant + dexamethasone + ondansetron, from part 2 (MEC regimen)

presence (regimen C) or absence (regimen B) of casopitant. Casopitant alone (regimen A) produced an AE frequency of $17 \%$ with no headache or dizziness (Table 4 ).

In part 2, the frequency of subjects with AEs following administration of casopitant plus IV dexamethasone and oral ondansetron (regimen F) was similar to the frequency of AEs with casopitant alone (regimen D) with $24 \%$ each and the number of AEs following administration of IV dexamethasone and oral ondansetron without casopitant (regimen E) was actually numerically higher (43\%) than the other two regimens (Table 4). No statistical analyses of the safety data were performed.

The majority of AEs were considered not related to study medication and were considered to be mild in nature. 
Table 4 Adverse events reported by two or more subjects

\begin{tabular}{|c|c|c|c|c|c|c|}
\hline \multirow[b]{3}{*}{ Adverse event } & \multicolumn{6}{|c|}{ Number of subjects (\%) } \\
\hline & \multicolumn{3}{|l|}{ Part 1} & \multicolumn{3}{|l|}{ Part 2} \\
\hline & $\begin{array}{l}\text { Regimen A } \\
(N=23)\end{array}$ & $\begin{array}{l}\text { Regimen B } \\
(N=22)\end{array}$ & $\begin{array}{l}\text { Regimen C } \\
(N=19)\end{array}$ & $\begin{array}{l}\text { Regimen D } \\
(N=21)\end{array}$ & $\begin{array}{l}\text { Regimen E } \\
(N=21)\end{array}$ & $\begin{array}{l}\text { Regimen F } \\
(N=21)\end{array}$ \\
\hline Headache & 0 & $5(22 \%)$ & $1(4 \%)$ & $2(10 \%)$ & $2(10 \%)$ & $2(10 \%)$ \\
\hline Dizziness & 0 & $6(26 \%)$ & $5(22 \%)$ & 0 & 0 & 0 \\
\hline Disorientation & 0 & $2(9 \%)$ & 0 & 0 & 0 & 0 \\
\hline Dyspepsia & 0 & 0 & $2(9 \%)$ & 0 & 0 & 0 \\
\hline Fatigue & 0 & $2(9 \%)$ & $1(4 \%)$ & 0 & 0 & 0 \\
\hline Hiccups & 0 & $2(9 \%)$ & $1(4 \%)$ & 0 & 0 & 0 \\
\hline Insomnia & 0 & $2(9 \%)$ & 0 & 0 & $1(5 \%)$ & 0 \\
\hline Paraesthesia & 0 & 0 & 0 & 0 & $2(10 \%)$ & 0 \\
\hline Tachycardia & 0 & $1(4 \%)$ & $2(9 \%)$ & 0 & 0 & 0 \\
\hline Back pain & $1(4 \%)$ & $1(4 \%)$ & $1(4 \%)$ & 0 & 0 & 0 \\
\hline Abdominal pain (upper) & 0 & 0 & 0 & 0 & $1(5 \%)$ & $1(5 \%)$ \\
\hline Diarrhea & 0 & 0 & 0 & $1(5 \%)$ & $1(5 \%)$ & 0 \\
\hline Myalgia & 0 & $1(4 \%)$ & $1(4 \%)$ & 0 & 0 & 0 \\
\hline Nasopharyngitis & 0 & 0 & $1(4 \%)$ & 0 & 0 & $1(5 \%)$ \\
\hline Vasovagal syncope & $1(4 \%)$ & 0 & 0 & $1(5 \%)$ & 0 & 0 \\
\hline Abdominal pain & 0 & 0 & 0 & $1(5 \%)$ & 0 & 0 \\
\hline
\end{tabular}

All AEs resolved. There were no deaths on the study. One subject, who experienced a serious adverse event of pulmonary embolism (considered by the investigator to be possibly related to study medication) 2 days after completing part 1 , withdrew from the study. Warfarin therapy was instituted and the $\mathrm{AE}$ resolved prior to hospital discharge. Follow-up assessments of this subject after completion of warfarin therapy show the subject has an underlying diagnosis of type II protein $\mathrm{C}$ deficiency where antigen levels are normal but activity is decreased.

\section{Discussion}

Part 1 of this study was designed to examine the potential pharmacokinetic interactions of the addition of casopitant to a corticosteroid $/ 5-\mathrm{HT}_{3}$ receptor antagonist regimen that would be used for the prevention of CINV resulting from HEC $[10,19]$. As data from earlier studies suggested some potential for casopitant to increase the exposure of coadministered oral dexamethasone, the three-drug combination of casopitant, dexamethasone, and ondansetron utilized a reduced-dose regimen of dexamethasone, with the objective of showing that this three-drug regimen (regimen $\mathrm{C}$ ) provided approximately the same dexamethasone exposure as a standard-dose two-drug regimen of dexamethasone and ondansetron (regimen B). Part 1 showed that casopitant exposure was modestly affected by coadministration with dexamethasone and ondansetron, with a $28 \%$ increase in exposure on day 1 and a $34 \%$ decrease in exposure on day 3. These changes in casopitant exposure when combined with repeat-dose dexamethasone would not be considered clinically important given the patient to patient variability in the pharmacokinetics of CYP3A substrates, like casopitant, where exposures can vary between subjects by as much as $50 \%$ to $80 \%$, or higher depending on the substrate [14]; in addition, a 3-day regimen of casopitant combined with repeat-dose oral dexamethasone has recently been shown to be effective in the prevention of CINV resulting from HEC [24].

Part 1 also demonstrated that the reduced-dose regimen of dexamethasone (from $20 \mathrm{mg}$ to $12 \mathrm{mg}$ on day 1 and from $8 \mathrm{mg}$ twice daily to $8 \mathrm{mg}$ once daily on days 2 and 3) was a suitable adjustment for the pharmacokinetic effect of casopitant on dexamethasone exposure. Although the three-drug combination resulted in a slightly lower dexamethasone exposure on day 1 when compared to the standard-dose two-drug regimen (17\% lower), the $24 \mathrm{~h}$ dexamethasone exposure on day 3 was similar to the standard regimen. These results indicate that the 3-day regimen of oral casopitant increases dexamethasone by $39 \%$ on day 1 and $108 \%$ on day 3 . The exposure of $32 \mathrm{mg}$ IV ondansetron was not affected by $150 \mathrm{mg}$ casopitant when used in this three-drug regimen and, therefore, no dose adjustment is necessary.

The dose regimens in part 2 were designed to evaluate the potential pharmacokinetic interactions of the addition of casopitant to an IV dexamethasone/oral ondansetron two- 
drug regimen for the prevention of CINV resulting from MEC $[10,19]$. In this case, no significant drug interactions were expected and therefore no a priori dose reductions were used in the study.

Again, casopitant exposures were only modestly affected by coadministration with $8 \mathrm{mg}$ IV dexamethasone and $8 \mathrm{mg}$ twice daily oral ondansetron on day 1 (16\% increase) and were not affected following 3 days of coadministration with oral ondansetron. Addition of casopitant to the two-drug regimen of IV dexamethasone and oral ondansetron resulted in a $21 \%$ increase in dexamethasone $\operatorname{AUC}(0-\infty)$, but no change in ondansetron exposure on day 1 or day 3 . These results confirm that no alteration in IV dexamethasone or oral ondansetron dose is necessary when coadministered with 1-day or 3-day regimens of casopitant for the prevention of MEC and this three-drug regimen has recently been shown to be effective in the prevention of CINV resulting from MEC [8]. The lack of interaction between single and repeat doses of oral casopitant and single or repeat-doses of oral doses of the $5-\mathrm{HT}_{3}$ antagonists dolasetron or granisetron has also recently been shown [1] suggesting that any of these $5-\mathrm{HT}_{3}$ receptor antagonists could be used in a three-drug regimen with casopitant for the prevention of CINV.

Coadministration of repeat oral doses of casopitant with IV ondansetron and oral dexamethasone, or with IV dexamethasone and oral ondansetron, in healthy subjects was generally well tolerated under the conditions of this study. One serious adverse event of severe pulmonary embolism occurred 2 days following completion of treatment in part 1 . This event was considered possibly related to treatment with study medication(s); however, follow-up assessments of this subject after the completion of warfarin therapy suggest type II protein $\mathrm{C}$ deficiency, an important risk factor for venous thromboembolism. It has been recommended that the subject be followed by a hematologist because of a need for life-long anticoagulation.

The three drug combinations of casopitant, dexamethasone, and ondansetron employed in the current study have been successfully utilized in phase III clinical trials, including study arms that tested a convenient single oral dose of $150 \mathrm{mg}$ casopitant on day 1 only and replacing the day $1150 \mathrm{mg}$ oral dose of casopitant with a $90 \mathrm{mg}$ IV infusion of casopitant as part of a 3-day IV/oral regimen [3, $8,11,24]$. These results demonstrate that any of these three-dosage regimens for casopitant can be added to existing two-drug regimens consisting of a corticosteroid and a $5-\mathrm{HT}_{3}$ receptor antagonist. In the case of regimens for the prevention of CINV that include repeat-doses of oral dexamethasone (e.g., CINV resulting from HEC), a reduction in the dose of dexamethasone from $20 \mathrm{mg}$ to $12 \mathrm{mg}$ on day 1 and from $8 \mathrm{mg}$ twice daily to $8 \mathrm{mg}$ once daily on days 2 and 3 may be employed to maintain a similar dexamethasone exposure to that of the standard two-drug regimen. If a single day 1 dose of $150 \mathrm{mg}$ casopitant is to be used for the prevention of CINV, only the day 1 dose of dexamethasone would need to be reduced, as no interaction of significance would be expected to occur in the day or days after single-dose casopitant administration (half-life of $17 \mathrm{~h}$, unpublished results). In addition, as supraproportional increases in dexamethasone exposures with increasing dose have not been observed [16], the observations in the current study would be applicable to a dexamethasone dose of $20 \mathrm{mg}$, i.e., a $40 \%$ higher exposure would be expected, and this increase (equivalent to a dexamethasone dose of $28 \mathrm{mg}$ ) may not be considered clinically relevant given the variability in exposure of CYP3A substrates [14]. When 1- or 3-day regimens of casopitant are to be added to singledose IV dexamethasone and repeat-dose ondansetron for the prevention of CINV (e.g., that resulting from MEC), no adjustment in dexamethasone dose is required. This study also showed that casopitant does not impact the pharmacokinetics of oral or IV ondansetron.

Acknowledgements The authors acknowledge the assistance of Sabrina Pagliarusco for the bioanalysis of plasma samples and WynneWords LLC in the preparation of this manuscript.

Open Access This article is distributed under the terms of the Creative Commons Attribution Noncommercial License which permits any noncommercial use, distribution, and reproduction in any medium, provided the original author(s) and source are credited.

\section{References}

1. Adams L, Johnson B, Zhang K, Yue L, Kirby L, Lebowitz P, Stoltz R (J Support Care Cancer, companion paper) Minimal impact of casopitant, a novel NK-1 antagonist, on the pharmacokinetics of dolasetron and granisetron doi:10.1007/s00520-008-0572-4

2. Arpornwirat W, Albert I, Hansen VL et al (2006) Multicenter, randomized, double-blind, ondansetron (ond)-controlled, dose-ranging, parallel group trial of the neurokinin-1 receptor antagonist (NK-1 RA) casopitant mesylate for chemotherapy-induced nausea/vomiting $(\mathrm{CINV})$ in patients (pts) receiving moderately emetogenic chemotherapy (MEC). J Clin Oncol 24:8512

3. Aziz Z, Arpornwirat W, Herrstedt J et al (2008) Phase III results for the novel neurokinin-1 (NK-1) receptor antagonist, casopitant: 3-day IV/oral dosing regimen for chemotherapy-induced nausea and vomiting $(\mathrm{CINV})$ in patients (Pts) receiving moderately emetogenic chemotherapy (MEC). J Clin Oncol 26:20512 doi:10.1200/ JCO.2008.16.8112

4. Cohen L, de Moor CA, Eisenberg P et al (2007) Chemotherapyinduced nausea and vomiting: incidence and impact on patient quality of life at community oncology settings. Support Care Cancer 15:497-503 doi:10.1007/s00520-006-0173-z

5. De Mulder PH, Seynaeve C, Vermorken JB et al (1990) Ondansetron compared with high-dose metoclopramide in prophylaxis of acute and delayed cisplatin-induced nausea and vomiting. A multicenter, randomized, double-blind, crossover study. Ann Intern Med 113:834-840 
6. Dixon CM, Colthup PV, Serabjit-Singh CJ et al (1995) Multiple forms of cytochrome P450 are involved in the metabolism of ondansetron in humans. Drug Metab Dispos 23:1225-1230

7. Gentile DM, Tomlinson ES, Maggs JL et al (1996) Dexamethasone metabolism by human liver in vitro metabolite identification and inhibition of 6-hydroxylation. J Pharmacol Exp Ther 277:105-112

8. Grunberg S, Aziz Z, Shaharyan A et al (2008) Phase III results of a novel neurokinin-1 (NK-1) receptor antagonist, casopitant: single oral and 3-day oral dosing regimens for chemotherapyinduced nausea and vomiting (CINV) in patients (Pts) receiving moderately emetogenic chemotherapy (MEC). J Clin Oncol 26:9540 doi:10.1200/JCO.2008.18.7559

9. Grunberg SM, Deuson RR, Mavros P et al (2004) Incidence of chemotherapy-induced nausea and emesis after modern antiemetics. Cancer 100:2261-2268 doi:10.1002/cncr.20230

10. Grunberg SM, Osoba D, Hesketh PJ et al (2005) Evaluation of new antiemetic agents and definition of antineoplastic agent emetogenicity - an update. Support Care Cancer 13:80-84 doi:10.1007/s00520-004-0718-y

11. Herrstedt J, Grunberg S, Rolski J et al (2008) Phase III results for the novel neurokinin-1 (NK-1) receptor antagonist, casopitant: single oral dosing regimen for chemotherapy-induced nausea and vomiting (CINV) in patients (Pts) receiving highly emetogenic chemotherapy (HEC). J Clin Oncol 26:9549

12. Hesketh PJ, Kris MG, Grunberg SM et al (1997) Proposal for classifying the acute emetogenicity of cancer chemotherapy. J Clin Oncol 15:103-109

13. Hesketh PJ (2004) Management of nausea and vomiting in cancer and cancer treatment. Jones \& Bartlett Publishers, Boston

14. Lamba JK, Lin YS, Schuetz EG et al (2002) Genetic contribution to variable human CYP3A-mediated metabolism. Adv Drug Deliv Rev 54:1271-1294 doi:10.1016/S0169-409X(02)00066-2

15. Liau CT, Chu NM, Liu HE et al (2005) Incidence of chemotherapy-induced nausea and vomiting in Taiwan: physicians' and nurses' estimation vs. patients' reported outcomes. Support Care Cancer 13:277-286 doi:10.1007/s00520-005-0788-5

16. Loew D, Schuster O, Graul EH (1986) Dose-dependent pharmacokinetics of dexamethasone. Eur J Clin Pharmacol 30:225-230 doi:10.1007/BF00614309
17. Marty M, Pouillart P, Scholl S et al (1990) Comparison of the 5hydroxytryptamine3 (serotonin) antagonist ondansetron (GR $38032 \mathrm{~F}$ ) with high-dose metoclopramide in the control of cisplatin-induced emesis. N Engl J Med 322:816-821

18. McCune JS, Hawke RL, LeCluyse EL et al (2000) In vivo and in vitro induction of human cytochrome P4503A4 by dexamethasone. Clin Pharmacol Ther 68:356-366 doi:10.1067/mcp.2000. 110215

19. Multinational Association of Supportive Care in Cancer (2008) Antiemetic subcommittee guidelines. http://www.mascc.org

20. National Comprehensive Cancer Network Clinical practice guidelines in oncology. http://www.NCCN.org

21. Neymark N, Crott R (2005) Impact of emesis on clinical and economic outcomes of cancer therapy with highly emetogenic chemotherapy regimens: a retrospective analysis of three clinical trials. Support Care Cancer 13:812-818 doi:10.1007/s00520-0050803-x

22. Roila F, Hesketh PJ, Herrstedt J (2006) Prevention of chemotherapy- and radiotherapy-induced emesis: results of the 2004 Perugia international antiemetic consensus conference. Ann Oncol 17:20 28 doi:10.1093/annonc/mdj936

23. Schnell FM (2003) Chemotherapy-induced nausea and vomiting: the importance of acute antiemetic control. Oncologist 8:187-198 doi:10.1634/theoncologist.8-2-187

24. Strausz J, Rolski J, Aziz Z et al (2008) Phase III results for the novel neurokinin-1 (NK-1) receptor antagonist, casopitant: 3-day IV/oral dosing regimen for chemotherapy-induced nausea and vomiting $(\mathrm{CINV})$ in patients (Pts) receiving highly emetogenic chemotherapy (HEC). J Clin Oncol 26:20586

25. Sun CC, Bodurka DC, Weaver CB et al (2005) Rankings and symptom assessments of side effects from chemotherapy: insights from experienced patients with ovarian cancer. Support Care Cancer 13:219-227 doi:10.1007/s00520-0040710-6

26. Warr DG, Hesketh PJ, Gralla RJ et al (2005) Efficacy and tolerability of aprepitant for the prevention of chemotherapyinduced nausea and vomiting in patients with breast cancer after moderately emetogenic chemotherapy. J Clin Oncol 23:28222830 doi:10.1200/JCO.2005.09.050 